\title{
Nanomole per Liter per Milligram per Day
}

National Cancer Institute

\section{Source}

National Cancer Institute. Nanomole per Liter per Milligram per Day. NCI Thesaurus.

Code C119457.

A unit of concentration equal to nanomoles per liter, divided by milligrams per day. 